\title{
The added value of CA125 normalization before interval debulking surgery to the chemotherapy response score for the prognostication of ovarian cancer patients receiving neoadjuvant chemotherapy for advanced disease
}

\author{
Wei-feng Liang ${ }^{1,2^{*}}$, Li-juan Wang ${ }^{2^{*}}$, Hui $\mathrm{Li}^{2}$, Chang-hao Liu ${ }^{2}$, Miao-fang $\mathrm{Wu}^{2}$ and Jing $\mathrm{Li}^{\circledR}$ \\ 1. Department of Gynecology and Obstetrics, Qilu Hospital (Qingdao), Cheeloo College of Medicine, Shandong University, Qingdao, 266035, People's Republic of China. \\ 2. Department of Gynecologic Oncology, Sun Yat-sen Memorial Hospital, Sun Yat-sen University, Guangzhou, 510120, People's Republic of China. \\ *These authors contributed equally to this work. \\ $\triangle$ Corresponding authors: Miao-fang Wu, MD, Department of Gynecologic Oncology, Sun Yat-sen Memorial Hospital, Sun Yat-sen University, 33 Yingfeng Road, \\ Guangzhou 510000, People's Republic of China, Tel: +86 020 34070211, Fax: +86 020 34071260, E-mail: wmiaofang@mail.sysu.edu.cn; Jing Li, MD, PhD, Department of \\ Gynecologic Oncology, Sun Yat-sen Memorial Hospital, Sun Yat-sen University, 33 Yingfeng Road, Guangzhou 510000, People's Republic of China, Tel: +86 02034078521 , \\ Fax: +86020 34071260, E-mail: lijing228@mail.sysu.edu.cn. \\ (0) The author(s). This is an open access article distributed under the terms of the Creative Commons Attribution License (https://creativecommons.org/licenses/by/4.0/). \\ See http://ivyspring.com/terms for full terms and conditions.
}

Received: 2020.09.01; Accepted: 2020.11.15; Published: 2021.01.01

\begin{abstract}
Objective: To investigate whether CA125 normalization following neoadjuvant chemotherapy (NACT) can complement the chemotherapy response system (CRS) in the prognostication of patients with tubo-ovarian high-grade serous carcinoma (HGSC).

Methods: In total, 118 HGSC patients who received NACT followed by interval debulking surgery (IDS) for FIGO stage IIIC-IV disease were included, and their clinical data were retrospectively reviewed. The primary endpoint was progression-free survival (PFS). Cox regression analysis was performed to identify predictors of PFS.

Results: Following NACT, CRS3 was noted in 35 patients $(29.7 \%)$, and CA 125 normalization $(\leq 35 \mathrm{U} / \mathrm{ml})$ was noted in 54 patients (45.8\%). Both CRS3 and CA125 normalization were identified as independent prognosticators of PFS. Combining these two factors, we stratified the 106 patients into three groups with different risks of recurrence: low-risk group (CRS3 + post-NACT CA125 $35 \mathrm{U} / \mathrm{ml} ; n=17,14.4 \%$ ), intermediate-risk group (CRS3 + post-NACT CA125>35 U/ml; $\mathrm{n}=19,16.1 \%)$ and high-risk group (CRS1-2; $n=82,69.5 \%$ ). The differences in PFS between the three groups were significant (log-rank test, $P<0.0001$ ). In Cox regression analyses, the new stratification method was found to have an independent prognostic effect.

Conclusion: Both the CRS system and the normalization of CA1 25 following NACT could reliably predict the risk of recurrence following primary treatment. The combination of the two factors refined the prognostic stratification of HGSC patients who were treated with NACT and IDS.
\end{abstract}

Key words: biomarkers, chemotherapy, gynecologic oncology, prognosis

\section{Introduction}

Ovarian cancer is the most lethal gynecological cancer with 52,100 new cases and 22,500 deaths estimated in China in 2015 [1]. Primary debulking surgery (PDS) followed by platinum-based chemotherapy is the standard treatment for ovarian cancer [2]. However, even among patients who have no evidence of disease following chemotherapy, $70 \%$ experience relapse within the subsequent three years [3]. Cytoreduction to no gross residual disease (R0 resection) can significantly improve the prognosis of ovarian cancer patients. Therefore, it is currently well established that the ultimate goal of PDS is R0 resection, and maximal surgical effort should be made to this [2]. Aggressive debulking surgery is morbid, with a $20-25 \%$ major complication rate and a $1-2 \%$ mortality rate [4-6]. Given this information, for patients with nonresectable disease or those who are considered unable to tolerate PDS, neoadjuvant 
chemotherapy (NACT) with interval debulking surgery (IDS) is an acceptable alternative [2,3]. Supporting evidence for this treatment comes from three randomized controlled trials (RCTs) that compared NACT-IDS with PDS and reported that the prognosis of patients treated with NACT-IDS was noninferior to that of patients treated with PDS [7-9]. However, these trials have been criticized because the progression-free survival (PFS), OS and optimal debulking rates were lower than those reported in previous studies [10]. Therefore, the use of NACT remains under debate.

In the 2019 European Society for Medical Oncology (ESMO) ovarian cancer guidelines, a three-tiered chemotherapy response score (CRS) system was recommended for patients receiving NACT to evaluate tumor response and predict prognosis [11]. Since its description, the CRS system has been independently assessed in many studies [12-17]. Currently, it is considered an accurate and highly reproducible method to predict survival outcomes for patients with tubo-ovarian high-grade serous carcinoma (HGSC) [11]. Recently, the HGSC CRS Collaborative Network validated the prognostic role of the CRS system in a real-world, heterogeneous study population [15]. Pooling individual patient data from 11 countries, the authors reported that CRS3 was independently associated with an improved prognosis. Based on the findings, the CRS system is proposed as a surrogate for both PFS and OS [15].

For HGSC patients who are treated with NACT-IDS, another important prognostic marker is CA125. Considering that the early normalization of CA125 is associated with improved oncological outcomes [18,19], we hypothesized that combining CA125 normalization and CRS would further refine the prognostic stratification of HGSC patients and identify patients who could gain the most from the NACT-IDS treatment modality.

\section{Patients and Methods}

This was a retrospective cohort study. The primary objective was to investigate whether the normalization of CA125 $(\leq 35 \mathrm{U} / \mathrm{ml})$ before IDS could add prognostic value to the CRS system to predict PFS in HGSC patients treated with NACT-IDS. The data were collected from two tertiary-referral university hospitals in China. The study protocol was approved by the Institutional Review Board (approval NO. SYSEC-KY-KS-2020-088). Patient charts were reviewed to identify those with International Federation of Gynecology and Obstetrics (FIGO) stages IIIC-IV ovarian, fallopian tube, or primary peritoneal HGSC who received NACT-IDS at either hospital during the period from January 2012 to
November 2019.

The decision to recommend PDS was made based on the likelihood of achieving R0 resection and patient tolerance to the surgery. A multidisciplinary team (MDT), including two experienced gynecological oncologists, one pathologist and one radiologist, assessed the possibility of $\mathrm{R} 0$ resection. Pre-NACT biopsy was obtained from all patients and reviewed by two pathologists to confirm the histological type. Patients who did not complete the primary treatment or had received chemotherapy at an outside institution were excluded from analysis. NACT regimens were largely platinum- and paclitaxel-based and reflected the standard protocols in practice guidelines during the study period. According to the Society of Gynecologic Oncology (SGO) and American Society of Clinical Oncology (ASCO) guidelines [20], patients with a response to NACT or stable disease underwent IDS after $\leq 3$ cycles of NACT. IDS was performed via a midline laparotomy by experienced gynecologic oncologists. For patients considered to have tumor progression during NACT, IDS was not considered; they were treated with second-line chemotherapy regimens and were excluded from the final analysis. All patients received a minimum of six cycles of chemotherapy, which included at least three cycles of adjuvant chemotherapy following IDS $[2,20]$. Serum levels of CA125 were routinely measured at diagnosis, before each cycle of chemotherapy and at IDS. Written informed consent was obtained from all patients.

The pathology slides of specimens from IDS were independently reviewed by two pathologists. CRS analysis was performed based on omental samples, which was in line with the International Collaboration on Cancer Reporting (ICCR) guidelines [21]. In the case of disputes concerning CRS classification, a unanimous agreement was reached after sample re-evaluation.

Patients were seen in routine follow-up every three months for two years after primary treatment, every six months for the following three years, and every year thereafter. Follow-up visits included a gynecologic examination and measurements of tumor markers. Surveillance ultrasonography examination and computed tomography (CT) and magnetic resonance imaging (MRI) scans were performed at the discretion of the gynecologic oncologist. PFS was defined as the interval from the date of the completion of primary treatment to the date of recurrence. OS was defined as the interval from the date of the completion of primary treatment to death.

We calculated descriptive statistics including medians and portions. PFS and OS were evaluated using the Kaplan-Meier method and compared with 
the log-rank test. Cox proportional hazard models (enter method) were fitted to estimate hazard ratios (HRs) and 95\% confidence intervals (95\% CIs) for PFS and OS. For multiple comparisons of survival curves, the Bonferroni adjustment was applied. All statistical tests were two-sided, and differences were considered significant at $P<0.05$. STATA 12.0 (Stata Press, College Station, TX, USA) and MedCalc 17.0 (MedCalc Software Ltd, Ostend, Belgium) were used for all analyses.

\section{Results}

\section{Patient characteristics}

A total of 118 patients were included in the final analysis. Table 1 shows the patient demographics and treatment characteristics. All 118 patients received three cycles of NACT. The median time interval between the last cycle of NACT and IDS was 25 days (range: 22 to 28 days). Before NACT, all patients were noted to have elevated levels of CA125 (median: $1218.9 \mathrm{U} / \mathrm{ml}$, range: 106.4 to $17354.0 \mathrm{U} / \mathrm{ml}$ ). Following $\mathrm{NACT}, \mathrm{CA} 125 \leq 35 \mathrm{U} / \mathrm{ml}$ was documented in 54
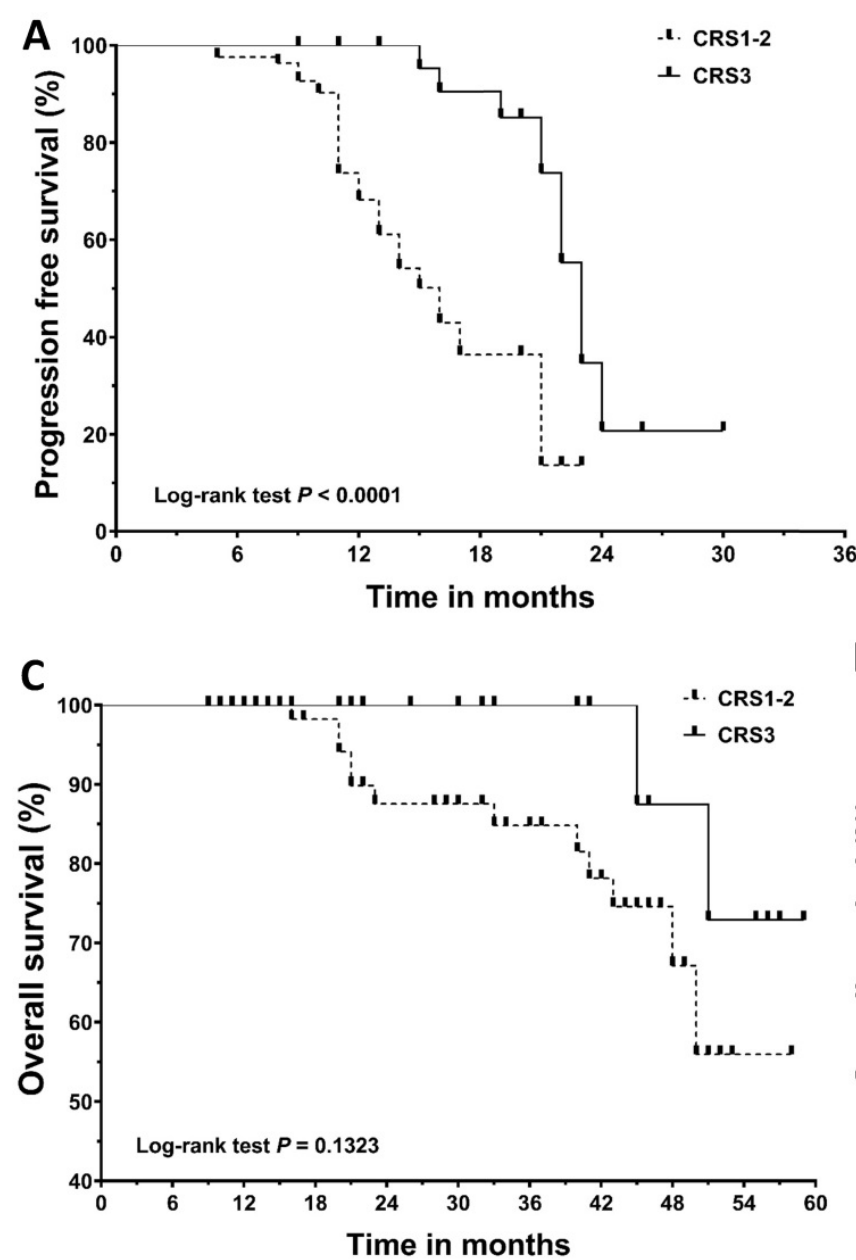

patients $(45.8 \%)$. Following NACT, CRS3 was noted in 35 patients $(29.7 \%)$. Of the 35 CRS3 patients, CA125 normalization was noted in $17(48.6 \%)$ patients. Supplementary Table 1 details CA125 status following NACT among CRS3 patients based on the presence or absence of complete pathological response (pCR). CA125 normalization was more frequently observed in CRS3 patients with pCR than CRS3 patients with near-complete response or minimally residual tumor $(72.7 \%$ vs. $37.5 \%)$; the difference was marginally significant $(P=0.053)$. All CRS3 patients underwent R0 cytoreduction in IDS and received three cycles of adjuvant chemotherapy after IDS. Of the 83 patients who did not achieve CRS3, 64 (77.1\%) underwent R0 debulking surgery, and $71(85.5 \%)$ received three cycles of chemotherapy following IDS.

\section{Survival outcomes stratified by CRS and CA125 normalization}

The median follow-up for the cohort was 21.0 months (range: 9 to 59 months). Recurrence and death were documented in 66 patients and 14 patients, respectively. Figure 1 shows the survival curves for
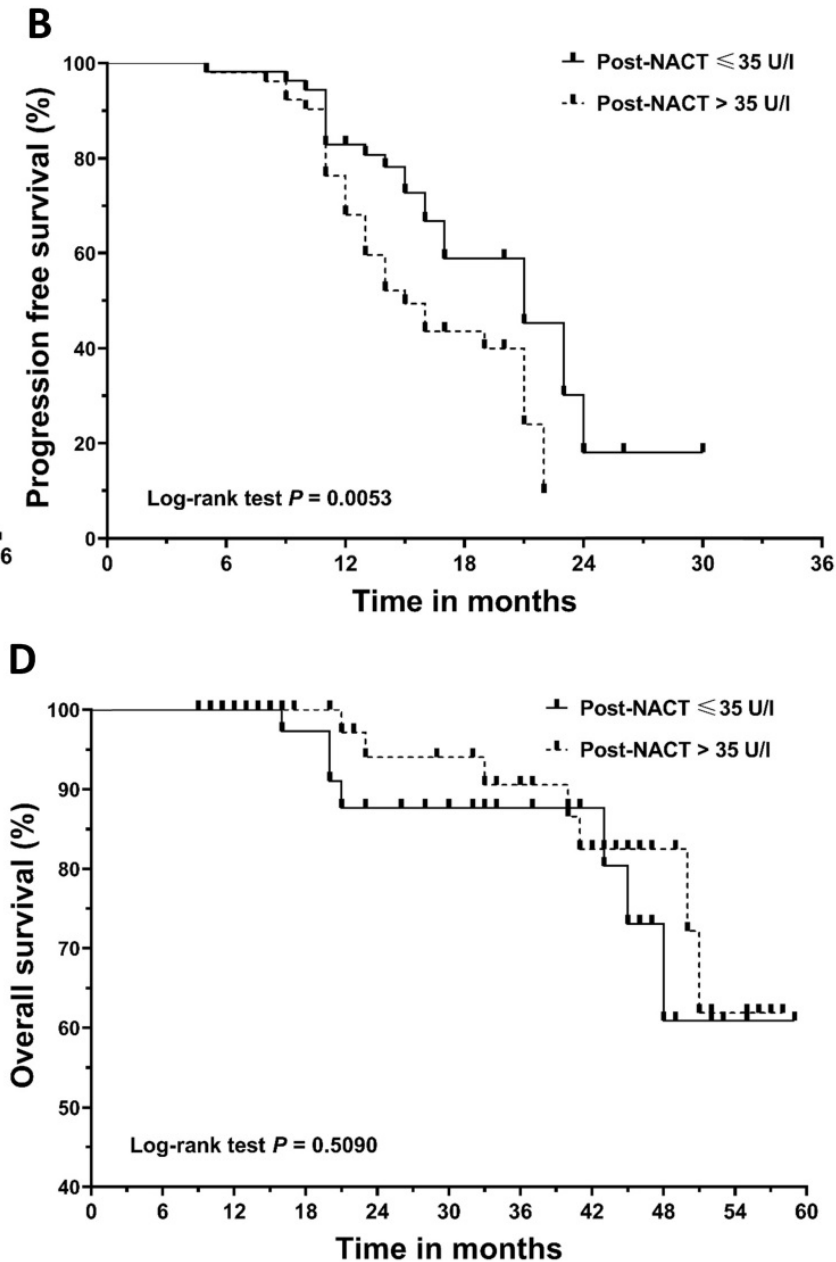

Figure 1. Kaplan-Meier curves for progression-free survival (PFS) and overall survival (OS). (A) PFS according to chemotherapy response score (CRS). (B) PFS according to post-NACT CA125 level. (C) OS according to chemotherapy response score (CRS). (D) OS according to post-NACT CA125 level. NACT, neoadjuvant chemotherapy. 
PFS and OS. CRS3 (HR=0.34; 95\% CI, 0.19 to 0.62; median PFS, 22 vs 16 months) and normalization of CA125 following NACT (HR $=0.51 ; 95 \% \mathrm{CI}, 0.30$ to 0.88 ; median PFS, 21 vs 16 months) were associated with a decreased risk of recurrence. Median OS was not achieved in our cohort. The difference in OS between the groups categorized by CRS3 (HR $=0.32$; 95\% CI, 0.07 to 1.45; median OS not achieved) and post-NACT CA125 (HR $=1.45 ; 95 \% \mathrm{CI}, 0.50$ to 4.15 ; median OS not achieved) did not reach significance. Table 2 summarizes the results of the univariate and multivariate Cox analyses. CRS3, normalization of CA125 following NACT and R0 resection were identified as independent prognosticators for PFS. Considering the importance of CA125 normalization, we conducted a subgroup analysis in patients who achieved CRS3 following NACT and the results are summarized in Table 3. After adjusting for other covariants, CA125 normalization was independently associated with a decreased risk of recurrence $(\mathrm{HR}=$ $0.08 ; 95 \% \mathrm{CI}, 0.02$ to $0.45 ; P=0.004)$.

\section{The added prognostic value of normalization of CA125 to CRS system}

Given the independent prognostic importance of CRS3 and the normalization of CA125 for PFS, we conducted further analysis to assess a new classification system by integrating the two factors. The cohort was divided into four subgroups: CRS3 + post-NACT CA125 $35 \mathrm{U} / \mathrm{ml}(\mathrm{n}=17,14.4 \%)$, CRS3 + post-NACT CA125 > $35 \mathrm{U} / \mathrm{ml}(\mathrm{n}=18,15.3 \%)$, CRS1-2 + post-NACT CA125 $\leq 35 \mathrm{U} / \mathrm{ml}(\mathrm{n}=37,31.4 \%)$ and CRS1-2 + post-NACT CA125 > $35 \mathrm{U} / \mathrm{ml}(\mathrm{n}=46$, $39.0 \%$ ). Figure $2 \mathrm{~A}$ demonstrates the survival curve for PFS; the differences between the four groups were significant (log-rank test, $P<0.0001$ ). Supplementary Table 2 summarizes the results of the post hoc Bonferroni analysis. Since the difference between the group with CRS1-2 + post-NACT CA125 $\leq 35 \mathrm{U} / \mathrm{ml}$ and the group with CRS1-2 + post-NACT CA125 > 35 $\mathrm{U} / \mathrm{ml}$ did not reach significance, we combined the two groups. Finally, using a combination of the CRS system and post-NACT CA125, we classified the 118 patients into three groups: low-risk group (CRS3 + post-NACT CA125 $35 \mathrm{U} / \mathrm{ml} ; \mathrm{n}=17,14.4 \%)$, intermediate-risk group (CRS3 + post-NACT CA125 > $35 \mathrm{U} / \mathrm{ml} ; \mathrm{n}=19,16.1 \%$ ) and high-risk group (CRS1-2; $n=82,69.5 \%$ ). Kaplan-Meier curves for PFS are shown in Figure 2B; the differences between the three groups were significant (log-rank test, $P<0.0001$ ). Table 4 summarizes the results of the post hoc Bonferroni analysis. The differences between the low-risk group and the intermediate-risk group (log-rank test, $P=$ 0.001 ), the low-risk group and the high-risk group (log-rank test, $P<0.0001$ ), and the intermediate-risk group and the high-risk group (log-rank test, $P=$ 0.040 ) were statistically significant. A Cox analysis was conducted where the low-risk group was used as a reference, and the results are shown in Table 5 . After adjusting for other prognosticators, the HRs for PFS of the intermediate-risk group and high-risk group were 4.54 (95\% CI, 1.47 to $14.00 ; P=0.008)$ and 6.10 (95\% CI, 2.14 to 17.40; $P=0.001)$, respectively.

Table 1. Baseline characteristics

\begin{tabular}{|c|c|}
\hline \multicolumn{2}{|l|}{ Variable } \\
\hline Age (years), median (range) & 58 (37 to 78$)$ \\
\hline BMI $\left(\mathrm{kg} / \mathrm{m}^{2}\right)$, median (range) & 22.3 (19.0 to 27.1$)$ \\
\hline \multicolumn{2}{|l|}{ FIGO stage, $\mathbf{n}(\%)$} \\
\hline IIIC & $104(88.1)$ \\
\hline IV & $14(11.9)$ \\
\hline \multicolumn{2}{|l|}{ ECOG performance status, $n(\%)$} \\
\hline Normal activity & $104(88.1)$ \\
\hline Restricted activity & $14(11.9)$ \\
\hline \multicolumn{2}{|l|}{ BRCA status including germline and somatic, $\mathbf{n}(\%)$} \\
\hline BRCA1 mutant & $3(2.5)$ \\
\hline BRCA2 mutant & $5(4.2)$ \\
\hline BRCA wide type & $8(6.8)$ \\
\hline Data unavailable & $102(86.4)$ \\
\hline \multicolumn{2}{|l|}{$\mathrm{R} 0$ resection, $\mathrm{n}(\%)$} \\
\hline Yes & $99(83.9)$ \\
\hline No & $19(16.1)$ \\
\hline \multicolumn{2}{|l|}{ ICU stay following IDS (\%) } \\
\hline Yes & $5(4.2)$ \\
\hline No & $113(95.8)$ \\
\hline \multicolumn{2}{|l|}{ NACT regimen (\%) } \\
\hline Three-week carboplatin/paclitaxel & $113(95.8)$ \\
\hline Weekly carboplatin/paclitaxel & $5(4.2)$ \\
\hline \multicolumn{2}{|l|}{ Chemotherapy response score } \\
\hline 1 & 55 (46.6) \\
\hline 2 & $28(23.7)$ \\
\hline 3 & $35(29.7)$ \\
\hline \multicolumn{2}{|l|}{ CA125 (U/ml), median (range) } \\
\hline Pre-NACT & $\begin{array}{l}1218.9 \text { (106.4 to } \\
17354.0)\end{array}$ \\
\hline Post-NACT & 38.4 (7.4 to 2292.0$)$ \\
\hline Normalization following NACT ( $\leq 35 \mathrm{U} / \mathrm{ml}), \mathrm{n}(\%)$ & $54(45.8)$ \\
\hline \multicolumn{2}{|l|}{ HE4 (pmol/l), median (range) } \\
\hline Pre-NACT & 616 (105 to 6397$)$ \\
\hline Post-NACT & 105 (19 to 1879$)$ \\
\hline \multicolumn{2}{|l|}{ HGB $(\mathrm{g} / \mathrm{l})$, median (range) } \\
\hline Pre-NACT & 98 (69 to 121$)$ \\
\hline Post-NACT & 106 (85 to 125$)$ \\
\hline \multicolumn{2}{|l|}{ Albumin (g/l), median (range) } \\
\hline Pre-NACT & 24 (16 to 37$)$ \\
\hline Post-NACT & 33 (26 to 41$)$ \\
\hline
\end{tabular}

\section{Discussion}

Whether NACT-IDS is noninferior to PDS regarding patient prognosis has been a controversial topic. Approximately $82 \%$ of SGO members did not consider that there is sufficient evidence to justify NACT [22]. Despite this, the utility of NACT in the United States increased from $7.7 \%$ in 2004 to $27.8 \%$ in 
2015 [23]. NACT can decrease tumor volume, thereby allowing for a less traumatic surgery and a higher R0 resection rate [20]. However, this treatment exposes a high tumor burden to chemotherapeutic drugs, which results in a selection of resistant tumor clones [24,25]. Even among HGSC patients with BRCA1heterozygous tumors that are supersensitive to DNAdamaging drugs and poly (ADP-ribose) polymerase (PARP) inhibitors, the utility of NACT is observed to facilitate the expansion of pre-existing BRCA1proficient tumor clones [26]. In addition, there have been clinical data suggesting that NACT patients are more likely to develop platinum-resistant recurrence $[27,28]$. The Japan Clinical Oncology Group phase III RCT 0602 (JCOG0602) compared NACT-IDS with PDS [29]. In 2020, the authors reported that the survival noninferiority of NACT was not validated in the trial [29]. At the Annual Meeting of ASCO in 2020, using data from the PAOLA-1 trial, C. Grimm et al. reported that among ovarian cancer patients receiving olaparib plus bevacizumab as maintenance therapy, the magnitude of PFS benefit was lower in NACT patients treated with NACT than in patients treated with PDS [30]. Collectively, these findings remind us of the potential negative influence of NACT. We believe that patients treated with NACT should be stratified according to their prognosis so they can have a better opportunity to gain a survival benefit from more individualized management.

Table 2. Univariate and multivariate progression-free survival analyses

\begin{tabular}{|c|c|c|c|c|c|c|}
\hline & \multicolumn{3}{|c|}{ Univariate analysis } & \multicolumn{3}{|c|}{ Multivariate analysis } \\
\hline & $\overline{\mathrm{HR}}$ & $95 \% \mathrm{CI}$ & $P$ value & $\mathrm{HR}$ & $95 \% \mathrm{CI}$ & $P$ value \\
\hline Age (Year) & 1.01 & 0.98 to 1.05 & 0.433 & & & \\
\hline $\begin{array}{l}\text { Stage (FIGO IIIC } \\
\text { vs IV) }\end{array}$ & 0.92 & 0.46 to 1.81 & 0.800 & & & \\
\hline $\operatorname{BMI}\left(\mathrm{kg} / \mathrm{m}^{2}\right)$ & 1.04 & 0.91 to 1.19 & 0.587 & & & \\
\hline $\begin{array}{l}\text { ECOG (Normal } \\
\text { activity vs } \\
\text { Restricted activity) }\end{array}$ & 1.30 & 0.68 to 2.48 & 0.430 & & & \\
\hline CRS3 (Yes vs No) & 0.34 & 0.19 to 0.62 & $<0.0001$ & 0.47 & 0.25 to 0.89 & 0.020 \\
\hline $\begin{array}{l}\text { Post-NACT } \\
\text { CA125<35 U/ml } \\
(\text { Yes vs No) }\end{array}$ & 0.51 & 0.30 to 0.88 & 0.015 & 0.49 & 0.28 to 0.84 & 0.010 \\
\hline $\begin{array}{l}\text { Post-NACT HE4 } \\
(\mathrm{pmol} / \mathrm{l})\end{array}$ & 1.00 & 0.9998 to 1.002 & 0.105 & & & \\
\hline $\begin{array}{l}\text { Post-NACT } \\
\text { albumin }(\mathrm{g} / \mathrm{l})\end{array}$ & 1.04 & 0.97 to 1.12 & 0.231 & & & \\
\hline $\begin{array}{l}\text { Post-NACT HGB } \\
(\mathrm{g} / \mathrm{l})\end{array}$ & 0.96 & 0.94 to 0.99 & 0.011 & 0.0 .99 & 0.96 to 1.02 & 0.663 \\
\hline ICU (Yes vs No) & 0.54 & 0.17 to 1.79 & 0.316 & & & \\
\hline $\begin{array}{l}\text { R0 resection in IDS } \\
\text { (Yes vs No) }\end{array}$ & 0.03 & 0.01 to 0.09 & $<0.0001$ & 0.04 & 0.01 to 0.12 & $<0.0001$ \\
\hline
\end{tabular}

The CRS system can divide HGSC patients who receive NACT according to their response: complete/near complete (CRS3), partial (CRS2), and no/minimal (CRS1) responses. Since the survival outcomes of the CRS1 patients were similar to those of the CRS2 patients, CRS can actually be treated as a binary prognostic system that stratifies patients into two subgroups [12,17]. Herein, we observed that CRS3 could be achieved in $29.7 \%$ of NACT patients, and this cohort had a significantly decreased risk of recurrence compared with those who achieved CRS1-2. These results are consistent with those of previous studies and confirmed that the CRS system can be used as a reliable tool for prognostic stratification $[15,17]$. In the study by the HGSC CRS Collaborative Network, more patients in the CRS3 group were noted to have a germline $B R C A 1 / 2$ mutation than those in the CRS1-2 group [15]. Therefore, CRS3 following NACT may suggest a favorable tumor biology, which provides a possible explanation for why CRS3 patients benefit more from NACT than the others.

Table 3. Multivariate progression-free survival analysis of patients achieving $\mathrm{R} 0$ resection in interval debulking surgery

\begin{tabular}{llll}
\hline & HR & $95 \%$ CI & $P$ value \\
\hline Age (Year) & 1.08 & 0.98 to 1.20 & 0.127 \\
Stage (FIGO IIIC vs IV) & 0.61 & 0.17 to 2.11 & 0.432 \\
BMI $\left(\mathrm{kg} / \mathrm{m}^{2}\right)$ & 1.41 & 0.92 to 2.17 & 0.115 \\
ECOG $($ Normal activity vs Restricted activity) & 0.47 & 0.04 to 5.12 & 0.539 \\
Post-NACT HE4 $(\mathrm{pmol} / \mathrm{l})$ & 1.00 & 0.99 to 1.01 & 0.625 \\
Post-NACT albumin $(\mathrm{g} / \mathrm{l})$ & 1.13 & 0.93 to 1.37 & 0.214 \\
Post-NACT HGB $(\mathrm{g} / \mathrm{l})$ & 1.01 & 0.94 to 1.09 & 0.738 \\
Post-NACT CA125 $(\mathrm{U} / \mathrm{ml})$ & 0.08 & 0.02 to 0.45 & 0.004 \\
ICU (Yes vs No) & 0.56 & 0.11 to 2.84 & 0.488 \\
\hline
\end{tabular}

BMI, body mass index; CI, confidence interval; CRS, chemotherapy response score; ECOG, eastern cooperative oncology group; FIGO, The International Federation of Gynecology and Obstetrics; HE4, Human epididymis protein 4; HGB, hemoglobin; $\mathrm{HR}$, hazard ratio; ICU, intensive care unit; NACT, neoadjuvant chemotherapy.

Table 4. Comparison of recurrence-free survival using the log-rank test with Bonferroni correction

\begin{tabular}{|c|c|c|c|c|c|c|}
\hline & \multicolumn{2}{|c|}{ Low-risk group } & \multicolumn{2}{|c|}{$\begin{array}{l}\text { Intermediate-risk } \\
\text { group }\end{array}$} & \multicolumn{2}{|c|}{ High-risk group } \\
\hline & Chi-Square & $P$ value & Chi-Square & $P$ value & Chi-Square & $P$ value \\
\hline Low-risk group & & & 11.422 & 0.001 & 15.389 & $<0.0001$ \\
\hline $\begin{array}{l}\text { Intermediate-risk } \\
\text { group }\end{array}$ & 11.422 & 0.001 & & & 4.203 & 0.040 \\
\hline High-risk group & 15.389 & $<0.0001$ & 4.203 & 0.040 & & \\
\hline
\end{tabular}

Table 5. Prognostic value of the combination of the chemotherapy response system and normalization of CA 125 for progression-free survival

\begin{tabular}{|c|c|c|c|c|c|c|}
\hline & $\begin{array}{l}\text { Unadjusted } \\
\text { HR }\end{array}$ & $95 \% \mathrm{CI}$ & $\begin{array}{l}P \\
\text { value }\end{array}$ & $\begin{array}{l}\text { Adjusted } \\
\mathrm{HR}^{\mathrm{a}}\end{array}$ & $95 \%$ CI & $\begin{array}{l}P \\
\text { value }\end{array}$ \\
\hline low-risk group & Reference & & & Reference & & \\
\hline $\begin{array}{l}\text { intermediate- } \\
\text { risk group }\end{array}$ & 4.20 & 1.39 to 12.72 & 0.011 & 4.54 & $\begin{array}{l}1.47 \text { to } \\
14.00\end{array}$ & 0.008 \\
\hline high-risk group & 7.40 & 2.70 to 20.23 & $\begin{array}{l}<0.00 \\
01\end{array}$ & 6.10 & $\begin{array}{l}2.14 \text { to } \\
17.40\end{array}$ & 0.001 \\
\hline
\end{tabular}

$\mathrm{CI}$, confidence interval; $\mathrm{HR}$, hazard ratio;

a Adjusted HRs for progression-free survival were adjusted for R0 resection in interval debulking surgery (Yes vs No) and post-NACT hemoglobin levels (g/l). 


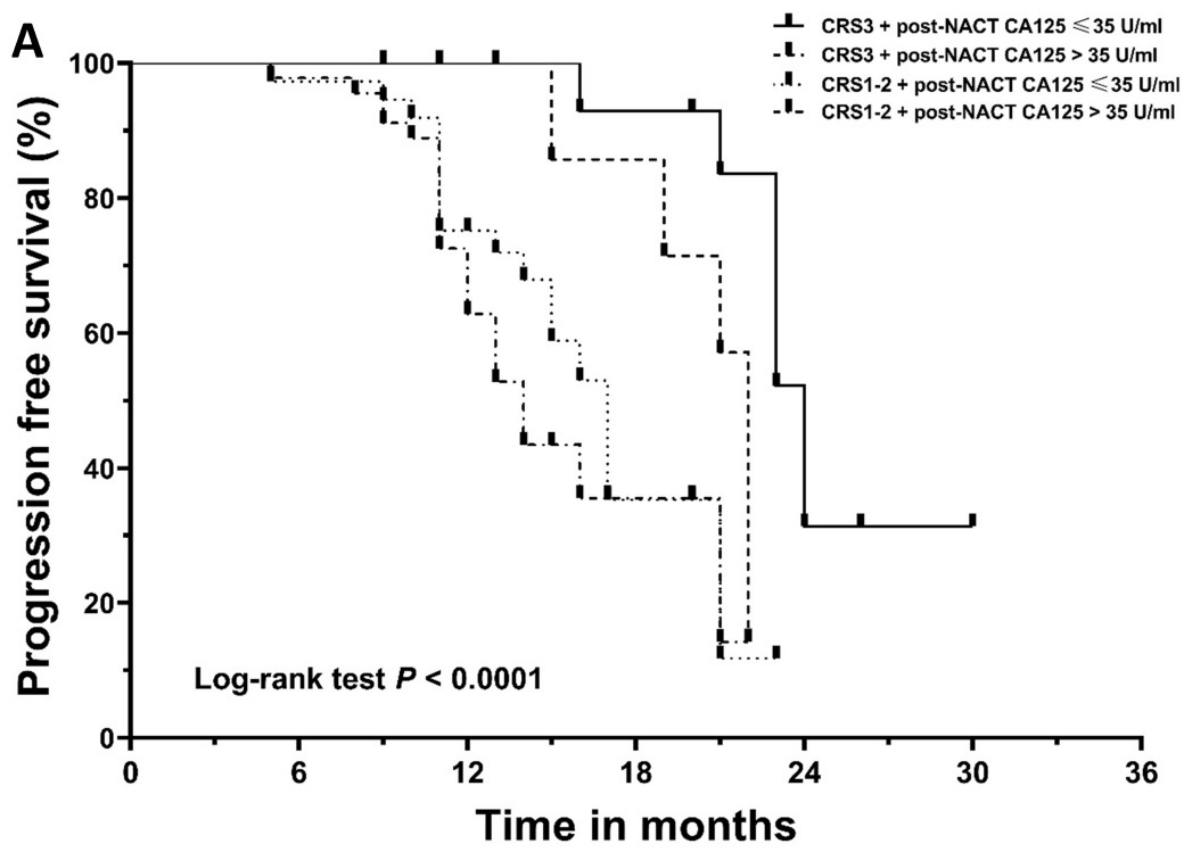

B

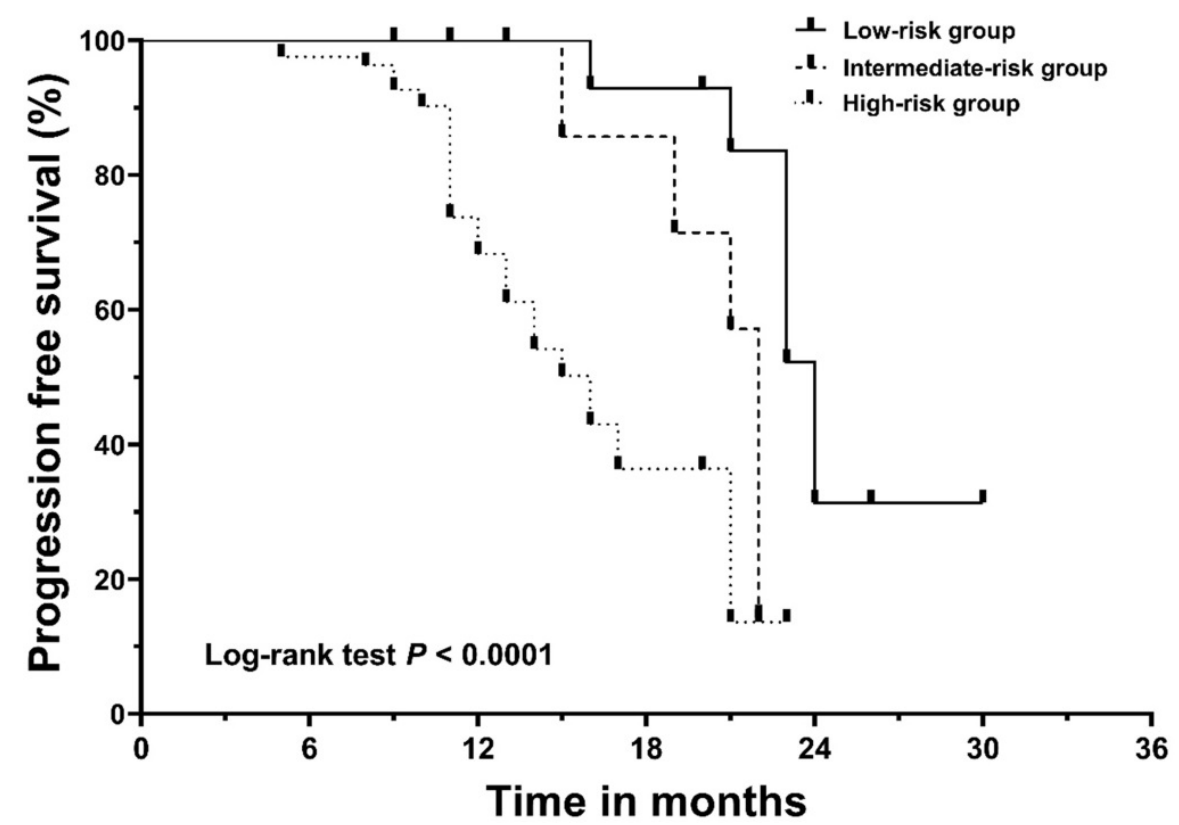

Figure 2. Kaplan-Meier curves for progression-free survival (PFS). (A) The patients were divided into four subgroups based on their chemotherapy response score (CRS) and post-NACT CA125 level. (B) The patients were stratified into low-, intermediate-, and high-risk categories. NACT, neoadjuvant chemotherapy.

In the neoadjuvant setting, CA125 can be utilized as a marker to assess the response to chemotherapy. The post-NACT CA125 level can predict the possibility of achieving optimal cytoreduction in IDS [31-33]. Despite this, the CA125 response does not exactly equate to the pathological response [12,15]. As we observed, CA125 normalization was noted in $48.6 \%$ of patients achieving CRS3. We identified the normalization of CA125 following NACT as an independent predictor of decreased recurrence risk.
Even in the CRS3 subgroup, patients with post-NACT CA125 $\leq 35 \mathrm{U} / \mathrm{ml}$ were observed to have a longer median PFS than those with post-NACT $>35 \mathrm{U} / \mathrm{ml}$, which was further confirmed in the Cox analysis. These findings were not completely consistent with those in previous studies [31,34-37]. However, given the following limitations in previous studies, caution must be taken in interpreting their results. First, many of the published studies included patients with nonserous epithelial cancer patients, yet the prognostic 
value of CA125 for these patients remains controversial [38]. Second, there is convincing evidence that NACT will exert a negative effect on patient prognosis if the number of NACT cycles exceeds four [37,39]. Although previous studies included patients receiving more than four cycles of NACT, the researchers did not consider or adjust the impact of the number of chemotherapy cycles. In light of these limitations, we believe that our findings complement those of prior studies and provide more reliable information regarding the prognostic role of CA125 normalization in NACT patients.

Since both the CRS system and the normalization of CA125 were independently associated with patient prognosis, we combined them and developed a new stratification method. Compared with the CRS system [12], the new method identified one more subgroup, the intermediate-risk group. Patients in the intermediate-risk group achieved CRS3 and received the same subsequent treatment as the other patients who achieved CRS3; however, they had a high risk of recurrence. The CRS system is based on omental assessment. Of note, the response of the omentum to NACT is not necessarily in line with that of other sites [12,15]. Therefore, CRS3 patients are a heterogeneous group with varying tumor loads. Since the CA125 level is correlated with tumor burden in $93 \%$ of ovarian cancer patients [40], it could help identify the cohort of patients with a higher tumor load among the CRS3 patients, thereby complementing the CRS system.

The present study is the first one to explore the role of combining the CRS system and the CA125 level in an attempt to refine the prognostic stratification of HGSC patients who were treated with NACT. Given that the CRS and CA125 are readily available in clinical practice, we believe that our findings are easily applicable in the care of patients in resourcelimited areas. Nevertheless, some limitations of the current study should be acknowledged. First, as a retrospective study, missing data could not be avoided, so the adjustment variables entered in the multivariable analyses might be incomplete. Second, since most health insurance plans in China do not cover the costs of genetic testing, information about $B R C A 1 / 2$ mutations was not available in most of the included patients, and we could not conduct a more detailed subgroup analysis. Third, although the PFS of our cohort was in line with that of previous reports $[12,14]$, the follow-up period in the present study was relatively short, and the median OS was not reached. Finally, the sample size of this work is limited. The data of the present study were collected from two tertiary-referral university hospitals in China. The clinical decisions in our institutions are made in accordance with the ASCO-SGO guidelines [20], and NACT has been prescribed to only carefully selected patients. Thus, although we pooled eight years of data, only 118 patients were included in the final analysis.

In conclusion, this study confirmed that the CRS system has prognostic significance. In addition, we found that CA125 normalization following NACT is an independent prognosticator, and it can provide complementary information to the CRS system for predicting the prognosis of HGSC patients who are treated with NACT. The combined use of the two methods could help physicians better stratify patients and make individualized therapeutic decisions. More studies are needed to validate these findings.

\section{Supplementary Material}

Supplementary tables.

http://www.jcancer.org/v12p0946s1.pdf

\section{Acknowledgements}

\section{Funding Statement}

This research was funded by China Scholarship Council (grant number 201906385061).

\section{Authors' contributions}

Data curation: WFL and LJW; Formal analysis: WFL, LJW and HL; Methodology: CHL; Conceptualization and visualization: MFW and JL; Writing - original draft: HL and MFW; Writing review \& editing: JL. All authors read and approved the final manuscript.

\section{Competing Interests}

The authors have declared that no competing interest exists.

\section{References}

1. Chen W, Zheng R, Baade PD, Zhang S, Zeng H, Bray F, et al. Cancer statistics in China, 2015. CA Cancer J Clin. 2016; 66: 115-32.

2. Gradishar WJ, Anderson BO, Abraham J, Aft R, Agnese D, Allison KH, et al. NCCN Clinical Practice Guidelines in Oncology (NCCN Guidelines ${ }^{\circledR}$ ). Journal of the National Comprehensive Cancer Network. 2020; 18.

3. Ledermann JA, Raja FA, Fotopoulou C, Gonzalez-Martin A, Colombo N, Sessa C, et al. Newly diagnosed and relapsed epithelial ovarian carcinoma: ESMO Clinical Practice Guidelines for diagnosis, treatment and follow-up. Ann Oncol. 2018; 29: iv259.

4. Chi DS, Zivanovic O, Levinson KL, Kolev V, Huh J, Dottino J, et al. The incidence of major complications after the performance of extensive upper abdominal surgical procedures during primary cytoreduction of advanced ovarian, tubal, and peritoneal carcinomas. Gynecol Oncol. 2010; 119: 38-42.

5. Gerestein CG, Nieuwenhuyzen-de Boer GM, Eijkemans MJ, Kooi GS, Burger CW. Prediction of 30-day morbidity after primary cytoreductive surgery for advanced stage ovarian cancer. Eur J Cancer. 2010; 46: 102-9.

6. Peiretti M, Zanagnolo V, Aletti GD, Bocciolone L, Colombo N, Landoni F, et al. Role of maximal primary cytoreductive surgery in patients with advanced epithelial ovarian and tubal cancer: Surgical and oncological outcomes. Single institution experience. Gynecol Oncol. 2010; 119: 259-64.

7. Kehoe S, Hook J, Nankivell M, Jayson GC, Kitchener H, Lopes T, et al. Primary chemotherapy versus primary surgery for newly diagnosed advanced ovarian cancer (CHORUS): an open-label, randomised, controlled, non-inferiority trial. Lancet. 2015; 386: 249-57. 
8. Vergote I, Trope CG, Amant F, Kristensen GB, Ehlen T, Johnson N, et al. Neoadjuvant chemotherapy or primary surgery in stage IIIC or IV ovarian cancer. N Engl J Med. 2010; 363: 943-53.

9. Fagotti A, Vizzielli G, Ferrandina G, Fanfani F, Gallotta V, Chiantera V, et al. Survival analyses from a randomized trial of primary debulking surgery versus neoadjuvant chemotherapy for advanced epithelial ovarian cancer with high tumor load (SCORPION trial). J Clin Oncol. 2018; 36, no. 15_suppl 5516.

10. Dizon DS. Neoadjuvant chemotherapy for newly diagnosed ovarian cancer: It's all about selection. Gynecol Oncol. 2017; 144: 241-2.

11. Colombo N, Sessa C, Du Bois A, Ledermann J, McCluggage W, McNeish I, et al. ESMO-ESGO consensus conference recommendations on ovarian cancer: pathology and molecular biology, early and advanced stages, borderline tumours and recurrent disease. Annals of Oncology. 2019; 30: 672-705.

12. Böhm S, Faruqi A, Said I, Lockley M, Brockbank E, Jeyarajah A, et al. Chemotherapy response score: development and validation of a system to quantify histopathologic response to neoadjuvant chemotherapy in tubo-ovarian high-grade serous carcinoma. Journal of Clinical Oncology. 2015; 33: $2457-63$

13. Lee JY, Chung YS, Na K, Kim HM, Park CK, Nam EJ, et al. External validation of chemotherapy response score system for histopathological assessment of tumor regression after neoadjuvant chemotherapy in tubo-ovarian high-grade serous carcinoma. J Gynecol Oncol. 2017; 28: e73.

14. Santoro A, Angelico G, Piermattei A, Inzani F, Valente M, Arciuolo D, et al. Pathological Chemotherapy Response Score in Patients Affected by High Grade Serous Ovarian Carcinoma: The Prognostic Role of Omental and Ovarian Residual Disease. Front Oncol. 2019; 9: 778.

15. Cohen PA, Powell A, Bohm S, Gilks CB, Stewart CJR, Meniawy TM, et al. Pathological chemotherapy response score is prognostic in tubo-ovarian high-grade serous carcinoma: A systematic review and meta-analysis of individual patient data. Gynecol Oncol. 2019; 154: 441-8.

16. Zorzato PC, Zannoni GF, Tudisco R, Pasciuto T, Di Giorgio A, Franchi M, et al. External validation of a 'response score' after neoadjuvant chemotherapy in patients with high-grade serous ovarian carcinoma with complete clinical response. Int J Gynecol Cancer. 2020; 30: 67-73.

17. Rajkumar S, Polson A, Nath R, Lane G, Sayasneh A, Jakes A, et al. Prognostic implications of histological tumor regression (Bohm's score) in patients receiving neoadjuvant chemotherapy for high grade serous tubal \& ovarian carcinoma. Gynecol Oncol. 2018; 151: 264-8.

18. Skaznik-Wikiel ME, Sukumvanich P, Beriwal S, Zorn KK, Kelley JL, Richard SD, et al. Possible use of CA-125 level normalization after the third chemotherapy cycle in deciding on chemotherapy regimen in patients with epithelial ovarian cancer: brief report. Int J Gynecol Cancer. 2011; 21: 1013-7.

19. Van Dalen A, Favier J, Burges A, Hasholzner U, de Bruijn H, Dobler-Girdziunaite D, et al. Prognostic significance of CA 125 and TPS levels after 3 chemotherapy courses in ovarian cancer patients. Gynecologic oncology. 2000; 79: 444-50.

20. Wright AA, Bohlke K, Armstrong DK, Bookman MA, Cliby WA, Coleman RL, et al. Neoadjuvant Chemotherapy for Newly Diagnosed, Advanced Ovarian Cancer: Society of Gynecologic Oncology and American Society of Clinical Oncology Clinical Practice Guideline. J Clin Oncol. 2016; 34: 3460-73.

21. INTEGRITY S. Ovary, Fallopian Tube and Primary Peritoneal Carcinoma Histopathology Reporting Guide.

22. Dewdney SB, Rimel BJ, Reinhart AJ, Kizer NT, Brooks RA, Massad LS, et al. The role of neoadjuvant chemotherapy in the management of patients with advanced stage ovarian cancer: survey results from members of the Society of Gynecologic Oncologists. Gynecol Oncol. 2010; 119: 18-21.

23. Meyer LA, Cronin AM, Sun CC, Bixel K, Bookman MA, Cristea MC, et al. Use and Effectiveness of Neoadjuvant Chemotherapy for Treatment of Ovarian Cancer. J Clin Oncol. 2016; 34: 3854-63.

24. Narod S. Can advanced-stage ovarian cancer be cured? Nat Rev Clin Oncol. 2016; 13: 255-61.

25. Zhang GN, Liu H, Huang JM, Wang L, Zhao JS, Li C, et al. TP53 K351N mutation-associated platinum resistance after neoadjuvant chemotherapy in patients with advanced ovarian cancer. Gynecol Oncol. 2014; 132: 752-7.

26. Sokolenko AP, Savonevich EL, Ivantsov AO, Raskin GA, Kuligina ES, Gorodnova TV, et al. Rapid selection of BRCA1-proficient tumor cells during neoadjuvant therapy for ovarian cancer in BRCA1 mutation carriers. Cancer Lett. 2017; 397: 127-32

27. Rauh-Hain JA, Nitschmann CC, Worley MJ, Jr, Bradford LS, Berkowitz RS, Schorge JO, et al. Platinum resistance after neoadjuvant chemotherapy compared to primary surgery in patients with advanced epithelial ovarian carcinoma. Gynecol Oncol. 2013; 129: 63-8.

28. Luo Y, Lee M, Kim HS, Chung HH, Song YS. Effect of neoadjuvant chemotherapy on platinum resistance in stage IIIC and IV epithelial ovarian cancer. Medicine (Baltimore). 2016; 95: e4797.

29. Onda T, Satoh T, Ogawa G, Saito T, Kasamatsu T, Nakanishi T, et al Comparison of survival between primary debulking surgery and neoadjuvant chemotherapy for stage III/IV ovarian, tubal and peritoneal cancers in phase III randomised trial. Eur J Cancer. 2020; 130: 114-25.

30. Grimm C, Cropet C, Ray-Coquard I. Maintenance olaparib plus bevacizumab (bev) after platinum-based chemotherapy plus bev in patients (pts) with newly diagnosed advanced high-grade ovarian cancer (HGOC): Efficacy by timing of surgery and residual tumor status in the Phase III PAOLA-1 trial. SGO 2020 Annual Meeting on Women's Cancer. 2020.
31. Pelissier A, Bonneau C, Chereau E, de La Motte Rouge T, Fourchotte V, Darai E, et al. CA125 kinetic parameters predict optimal cytoreduction in patients with advanced epithelial ovarian cancer treated with neoadjuvant chemotherapy. Gynecol Oncol. 2014; 135: 542-6.

32. Zeng J, Yin J, Song X, Jin Y, Li Y, Pan L. Reduction of CA125 Levels During Neoadjuvant Chemotherapy Can Predict Cytoreduction to No Visible Residual Disease in Patients with Advanced Epithelial Ovarian Cancer, Primary Carcinoma of Fallopian tube and Peritoneal Carcinoma. J Cancer. 2016; 7: 2327-32

33. Pelissier A, Roulot A, Guery B, Bonneau C, Bellet D, Rouzier R. Serum CA125 and HE4 levels as predictors for optimal interval surgery and platinum sensitivity after neoadjuvant platinum-based chemotherapy in patients with advanced epithelial ovarian cancer. J Ovarian Res. 2016; 9: 61.

34. Le T, Faught $\mathrm{W}$, Hopkins L, Fung-Kee-Fung M. Importance of CA125 normalization during neoadjuvant chemotherapy followed by planned delayed surgical debulking in patients with epithelial ovarian cancer. J Obstet Gynaecol Can. 2008; 30: 665-70.

35. Furukawa N, Sasaki Y, Shigemitsu A, Akasaka J, Kanayama S, Kawaguchi R, et al. CA-125 cut-off value as a predictor for complete interval debulking surgery after neoadjuvant chemotherapy in patients with advanced ovarian cancer. J Gynecol Oncol. 2013; 24: 141-5.

36. Vallius T, Hynninen J, Auranen A, Carpen O, Matomaki J, Oksa S, et al. Serum HE4 and CA125 as predictors of response and outcome during neoadjuvant chemotherapy of advanced high-grade serous ovarian cancer. Tumour Biol. 2014; 35: 12389-95.

37. Xu X, Deng F, Lv M, Chen X. The number of cycles of neoadjuvant chemotherapy is associated with prognosis of stage IIIc-IV high-grade serous ovarian cancer. Arch Gynecol Obstet. 2017; 295: 451-8.

38. Stenman UH, Alfthan H, Vartiainen J, Lehtovirta P. Markers supplementing CA 125 in ovarian cancer. Ann Med. 1995; 27: 115-20.

39. Bristow RE, Chi DS. Platinum-based neoadjuvant chemotherapy and interval surgical cytoreduction for advanced ovarian cancer: a meta-analysis. Gynecol Oncol. 2006; 103: 1070-6.

40. Bast RC, Jr., Klug TL, St John E, Jenison E, Niloff JM, Lazarus H, et al. A radioimmunoassay using a monoclonal antibody to monitor the course of epithelial ovarian cancer. N Engl J Med. 1983; 309: 883-7. 